\title{
El desarrollo de la ciencia geográfica en América Latina. La producción científica a través de Revista de Geografía Norte Grande ${ }^{1}$
}

\author{
Rodrigo Hidalgo ${ }^{2}$, Rafael Sánchez ${ }^{3}$, Daniel Santana ${ }^{4}$ y Federico Arenas ${ }^{5}$
}

\section{RESUMEN}

Al igual que el resto de las ciencias, la geografía ha transitado por diferentes etapas en las últimas décadas. Revista de Geografía Norte Grande (RGNG) ha canalizado y difundido las más importantes investigaciones de la ciencia geográfica producidas en América Latina. En este artículo se indaga en lo que ha sido publicado en este medio de comunicación, para ello se construyó una base de datos con datos de todos los artículos publicados entre 1974 y 2014, y la información se analizó a partir de tres periodos: 1974-1990, 1991-2005 y 2006-2014. Se logró identificar como la revista de carácter local se consolida, para luego sufrir una apertura geográfica y temática progresiva, así como también determinar el quehacer y pensamiento de la ciencia geográfica durante este periodo, evaluar las consecuencias del trabajo realizado y proyectar las nuevas preguntas que trazarán del devenir de esta disciplina.

Palabras clave: Geografía, publicaciones científicas, teoría y métodos de la geografía.

\begin{abstract}
Just as is the case for other sciences, geography has transitioned in the last few decades. The Revista Geografía Norte Grande (RGNG) has channeled and disseminated the most important research in geographical science produced in Latin America. In order to explore the work published in this journal we created a database containing details of all articles published from 1974-2004. Data was analyzed for three periods: 1974-1990, 1991-2005 and 2006-2014. This analysis made it possible to identify the initial local nature of the journal, which then opened to more progressive geographic themes. We were also able to determine the type of geographic research and thought during these periods, in order to evaluate the consequences of the work undertaken and project the new questions that will define the evolution of this discipline.
\end{abstract}

Key words: Geography, scientific publications, theory and methods of geography.

1 Artículo recibido el 28 de diciembre de 2014, aceptado el 16 de enero de 2015 y corregido el 6 de febrero de 2015.

2 Instituto de Geografía, Pontificia Universidad Católica de Chile (Chile). E-mail: rhidalgd@uc.cl
3 Instituto de Geografía, Pontificia Universidad Católica de Chile (Chile). E-mail: rsanchez@uc.cl

4 Instituto de Geografía, Pontificia Universidad Católica de Chile (Chile).E-mail: Idsantana@uc.cl

5 Instituto de Geografía, Pontificia Universidad Católica de Chile (Chile). E-mail: farenasv@uc.cl 
La etapa final de una investigación científica, corresponde a la divulgación del conocimiento adquirido y la elección del medio adecuado, un hecho fundamental para conseguir la mayor difusión y validación entre los pares. En esta fase, las revistas científicas juegan un rol fundamental, pues constituyen el vehículo a través del cual se dan a conocer los más relevantes avances de cada una de las disciplinas, así como también la exposición y corrección de posibles errores de una investigación (Gangas y Santis, 1981; Ancker, 2003). Debido a esto, las revistas científicas constituyen un reservorio de las interrogantes fundamentales que posee una sociedad en un momento determinado de su existencia y las respuestas que los miembros de la misma lograron obtener, por tanto, un acucioso análisis de los contenidos de una publicación seriada permiten establecer una cronología del avance, innovación y/o declive que una ciencia en particular experimenta (Manríquez, 2001-2002).

Se considera que en el año 1665 aparecen las primeras revistas de carácter académico, la francesa Journal des Sçavans y la inglesa Philosophical Transactions of the Royal Society of London. A partir de ese momento, la proliferación de publicaciones que reúnen trabajos científicos se han multiplicado y especializado rápidamente (Abadal y Rius, 2006). En el caso latinoamericano, la primera revista científica correspondió al Mercurio Volante, que era una publicación dedicada a divulgar casos de estudios relacionados con la medicina en 1772 (López-Espinosa, 2000), a la cual posteriormente se le unieron publicaciones de las más diversas temáticas en cada uno de los nuevos países americanos. En la ciencia geográfica producida en América Latina y el Caribe, la revista más importante, considerando los actuales parámetros bibliométricos, corresponde a Revista de Geografía Norte Grande (RGNG), la cual este año publica su sexagésimo número. A pesar que no corresponde a la publicación vigente más antigua, sí constituye la que ha logrado mantener su publicación de manera ininterrumpida desde 1974 y ha accedido a las más importantes bases de datos y catálogos científicos existentes ${ }^{6}$. A lo largo de estos años,

\footnotetext{
6 Las publicaciones de geografía vigentes más antiguas de Chile (país de origen de RGNG) e incluso
}

la revista ha publicado más de 600 artículos de las más diversas áreas de la geografía y cuyos autores provienen de América, Europa y Asia. Si bien a lo largo de este periodo se han realizado interesantes reflexiones en torno a las temáticas que han sido publicadas (Brignardello, 1996), se han omitido una serie de datos que podrían contribuir a una mejor comprensión sobre el desarrollo de la ciencia geográfica en Chile y América Latina.

Los momentos claves de una institución otorgan un momento idóneo para realizar evaluaciones del trabajo realizado, tanto al interior de las unidades patrocinantes, los equipos que las dirigen y la visión que se proyecta hacia la sociedad. Debido a lo anterior, es posible señalar que RGNG ha canalizado y difundido las más importantes investigaciones de la ciencia geográfica producidas en América Latina y por lo tanto, indagar en lo que ha sido publicado en este medio de comunicación a lo largo de su existencia, es una herramienta válida para adentrarse en el quehacer y pensamiento de la ciencia geográfica en las últimas cuatro décadas, evaluar las consecuencias del trabajo realizado y proyectar las nuevas preguntas que trazarán del devenir de esta disciplina.

\section{La ciencia geográfica en América Latina}

Si bien la inversión en Ciencia y Tecnología en América Latina y el Caribe todavía se encuentra lejana de los grandes centros de desarrollo, los países poco a poco adquieren conciencia del valor que tiene destinar recursos económicos a la investigación de alta calidad. El gasto en relación al Producto Interno Bruto (PIB) ha crecido de manera lenta, pero constante. Mientras que en 1990 se destinaba el 0,48\% en el año 2012 se alcanzó, en

de América Latina y el Caribe corresponden a Revista de Geográfica de Chile Terra Australis, la cual se publicó por primera vez en septiembre de 1948 como medio de divulgación del Comité Nacional de Geografía, Geodesia y Geofísica; e Investigaciones Geográficas del Departamento de Geografía de la Universidad de Chile que fue creada en el año 1951 bajo el nombre de "Informaciones Geográficas". Sin embargo, ambas revistas han visto interrumpida en reiteradas ocasiones su publicación (Zapater et al., 1978; Manríquez, 2001-2002). 
promedio, el 0,75\%. Lo anterior ha permitido incrementar el número de universidades y centros de formación técnica, elevar el número de titulados en la educación superior, crear nuevos programas de licenciatura, maestrías y doctorados, aumentar el número de becas y créditos para estudios de postgrado, crear instituciones públicas y privadas de apoyo a la ciencia y la tecnología, entre otros aspectos. Esto ha permitido, por ejemplo, que el número de graduados como doctores pasara de 532 en 1990 a más de 22.469 en el 2011, y que el número de publicaciones científicas de alto impacto registrados en indicadores como Science Citation Index $(\mathrm{SCl})$ se incrementara desde 11.046 en 1990 hasta los 70.084 artículos en el 2011 (RICYT, 2015).

Tal como variada es la biodiversidad latinoamericana, heterogéneo ha sido el porvenir de la investigación científica y el desarrollo en cada uno de los países. Mientras que Brasil constituye la única nación que ha decidido destinar más de un punto porcentual de su PIB a la ciencia y tecnología, Ilegando a fines de la primera década del siglo XXI a 1,23\% de su PIB, Guatemala y El Salvador reservan solo el $0,04 \%$ y $0,03 \%$ respectivamente. Esta diferencia en los países se refleja rápidamente -aunque no de manera directa- en indicadores como el número de publicaciones por cada 100.000 habitantes donde Argentina muestra 21,84 artículos y Perú solo 2,66 para el año 2011; o en el número de patentes solicitadas por residentes o no residentes en cada país, donde Uruguay presenta 688 y Colombia 1.981 (RICYT, 2015).

A una mayor escala también se vuelve más interesante esta diversidad. Según datos disponibles de la Red de Indicadores de Ciencia y Tecnología -lberoamericana e Interamericana- (RICYT) para el año 2011, a nivel de disciplinas científicas el gasto en I+D muestra una fuerte tendencia por la búsqueda y desarrollo de tecnologías que permitan un manejo práctico y eficiente de los recursos económicos existentes para beneficio de la sociedad, en desmedro de las disciplinas denominadas como humanidades. De esta manera, las ingenierías concentran más del $23 \%$ de los recursos en I+D, destacando en Argentina con un $37,15 \%$ del gasto involucrado. A las ciencias agrícolas se destina el $18 \%$, alcanzando el $36,92 \%$ en Paraguay. En tercer lugar, se en- cuentran las ciencias naturales y exactas que atraen un $17 \%$ del gasto, con un máximo de $26,21 \%$ en Uruguay, pero solo un 5,59\% en Paraguay. Por su parte, las ciencias sociales reúnen un $16 \%$ del gasto promedio, alcanzando notablemente el $48,61 \%$ en El Salvador y $24,02 \%$ en Guatemala. Por su parte, las ciencias médicas absorben el $11 \%$ del gasto, cifra que se eleva a $34,38 \%$ en Guatemala, reduciéndose a solo un 1,47\% en Ecuador. Finalmente, a las humanidades corresponden a las disciplinas a las que menos se les destina gasto en $\mathrm{I}+\mathrm{D}$ con un promedio de 3,50\%.

En el caso de la ciencia geográfica, su desarrollo en Latinoamérica ha evolucionado conforme a lo ocurrido con el resto de las ciencias sociales. Desde una posición destacada a mediados del siglo XIX hasta una constante pérdida de presencia tanto con el debate público como en los programas de educación formal en los inicios del siglo XXI (Asociación Americana de Geógrafos, 2006). Lo curioso de esta situación es que ha ocurrido de manera opuesta al proceso de institucionalización, profesionalización y especialización que ha experimentado la disciplina, y que se expresa en la apertura de numerosos departamentos e Institutos de Geografía (tanto gubernamentales como académicos); la constante creación de programas de licenciatura, magíster y doctorado, los cuales para el año 2011 se estimaban en un total de 181 (Palacio Prieto, 2011); el fortalecimiento y consolidación de redes y organizaciones geográficas, tanto al interior de América Latina como a nivel global, tales como el Instituto Panamericano de Geografía e Historia y Unión Geográfica Internacional; el incremento de la publicación de artículos en revistas y libros de corriente principal; y una activa participación y organización de encuentros científicos internacionales.

Un punto destacado lo constituye la calidad de la producción de la ciencia geográfica en esta región. Latinoamérica siempre se ha caracterizado por poseer una larga tradición en la creación de publicaciones de divulgación científica y académica. Sin embargo, esta significativa fertilidad literaria no era acompañada por protocolos objetivos y medibles. Era muy común observar boletines que reunían las reflexiones de geógrafos sobre temas contingentes, informaban sobre 
encuentros o eventos científicos o bien, canalizaban las investigaciones de los académicos de la misma institución editora, situación que reflejaba una excesiva endogamia, carencia de procesos de evaluaciones transparentes, inexistencia de equipos editoriales permanentes e inestables fechas de publicación. Con estas características las revistas, incluso las más tradicionales, tenían un perfil poco internacional (Gutiérrez, 1999).

Sin embargo, a partir del advenimiento del nuevo siglo muchas publicaciones comienzan a experimentar transformaciones tendientes a eliminar las malas prácticas, adoptando estándares de calidad más objetivos e internacionales. Este proceso es impulsado y acompañado por distintas redes de cooperación regional en materia de publicaciones, tales como el Sistema Regional de Información para las Revistas Científicas de
América Latina, el Caribe, España y Portugal (Latindex); la Red de Revistas Científicas de América Latina y el Caribe, España y Portugal (redalyc); y la Scientific Electronic Library Online (SciELO). De esta manera, en la actualidad las revistas de geografía cumplen gran parte de los criterios exigidos para las publicaciones de carácter científico, así lo reflejan las cerca de 240 revistas de geografía y de temas afines registradas en Latindex.

A partir de esta estandarización de procesos y mecanismos de las publicaciones científicas, algunas revistas lograron acceder a bases de datos y catálogos de mayor prestigio y exigencia. Del total de revistas existentes en América Latina, cinco de ellas se encuentran indexadas en Scopus de Elsevier (Cuadro $\mathrm{N}^{\circ}$ 1) y una en Web of Science (Thomson Reuters, ex ISI), la Revista de Geografía Norte Grande desde 2009.

\section{Cuadro $\mathrm{N}^{\circ} 1$}

Posición de revistas de geografía de América Latina indexadas en SCImago (subcategoría Geografía, Planificación y Desarrollo), 2013

\begin{tabular}{|c|c|c|c|c|c|}
\hline Título & País & Institución editora & $\begin{array}{l}\text { Año } \\
\text { inicio }\end{array}$ & $\begin{array}{l}\text { Año de in- } \\
\text { dexación } \\
\text { en Scopus }\end{array}$ & $\begin{array}{l}\text { Índice de } \\
\text { Impacto } \\
\text { en Scopus }\end{array}$ \\
\hline $\begin{array}{l}\text { Revista de Geografía } \\
\text { Norte Grande }\end{array}$ & Chile & $\begin{array}{l}\text { Instituto de Geografía, } \\
\text { Pontificia Universidad } \\
\text { Católica de Chile }\end{array}$ & 1974 & 2005 & 0,148 \\
\hline $\begin{array}{l}\text { RA'E GA - O Espaco } \\
\text { Geografico em Analise }\end{array}$ & Brasil & $\begin{array}{l}\text { Universidad Federal de } \\
\text { Paraná }\end{array}$ & 1997 & 2003 & 0,110 \\
\hline $\begin{array}{l}\text { Investigaciones } \\
\text { Geográficas: Boletín del } \\
\text { Instituto de Geografía }\end{array}$ & México & $\begin{array}{l}\text { Instituto de Geografía, } \\
\text { Universidad Nacional, } \\
\text { Autónoma de México }\end{array}$ & 1969 & 2002 & 0,107 \\
\hline Caribbean Geography & Jamaica & $\begin{array}{l}\text { University of the West } \\
\text { Indies }\end{array}$ & 1983 & 1999 & 0,101 \\
\hline $\begin{array}{l}\text { Revista Geográfica } \\
\text { Venezolana }\end{array}$ & Venezuela & $\begin{array}{l}\text { Instituto de Geografía } \\
\text { y Conservación de } \\
\text { Recursos Naturales, } \\
\text { Universidad de los } \\
\text { Andes }\end{array}$ & 1959 & 1999 & 0,101 \\
\hline
\end{tabular}

Fuente: Journal Rankings, SCImago, 2013

Este acceso a bases de datos y catálogos internacionales ha permitido que la geografía proveniente de Latinoamérica, poco a poco, alcance mayor visibilidad y logre ser recono- cida en el mundo científico. Según datos de SCImago, entre 1996 y 2013 el número de documentos pertenecientes a la categoría de las Ciencias Sociales y, específicamente a la 
subcategoría Geografía, Planificación y Desarrollo, y que han sido publicadas en revistas provenientes de la región latinoamericana corresponden a 8.259 artículos. Si en 1996 se publicaron 136 trabajos, 17 años después las principales revistas de América Latina publicaron 908. Si bien, estos datos no implican, necesariamente, que los autores de estos artículos se encuentren afiliados a alguna institución en América Latina, sí permiten demostrar que en otras partes del mundo sí se accede y han adquirido prestigio las revistas editadas en esta región, convirtiéndose en una alternativa para la publicación de trabajos de alta calidad.

\section{La Revista de Geografía Norte Grande}

El primer número de RGNG fue publicado en 1974. El objetivo era dar a conocer al resto de la comunidad científica los resultados de las investigaciones obtenidas por el grupo de especialistas pertenecientes al Taller del Norte Grande del recién formado Instituto de Geografía de la Universidad Católica de Chile. Debido a esta razón, la publicación fue bautizada como Revista Norte Grande.

Para realizar el análisis se construyó una base de datos con todos los artículos publicados en RGNG entre 1974 y 2014, y la información obtenida se dividió en tres periodos: 19741990, 1991-2005 y 2006-2014, los cuales permitieron visualizar y realizar un seguimiento a las principales características de la ciencia geográfica en las últimas cuatro décadas.

\section{4-1990: la consolidación de una geografía local que mira hacia Europa}

Revista de Geografía Norte Grande surgió en un momento clave de la historia contemporánea: por un lado, los modelos de desarrollo keynesiano perecían bajo el impulso de una crisis económica de carácter sistémico y por otro, en Chile daba inicio el más temprano de los experimentos neoliberales. Producto del primer hecho, la legitimidad social de la geografía neopositivista comenzaba a ser cuestionada por las primeras críticas radicales, que aunque variadas y poco rigurosas teóricamente (Peet, 2012), Ilegarían a convertirse en un discurso dominante en el contexto anglosajón (Delgado, 2003), mientras que en los contextos de Francia y Alemania la geografía regional comenzaba a evolucionar hacia la geografía activa (Moreira, 2008) en el primer caso y de manera más acelerada hacia la ciencia regional en el segundo (Bahrenberg, 1979). En América Latina, los procesos de institucionalización desde afuera de la disciplina geográfica comienzan en la década de 1950 gracias al arribo de una oleada de geógrafos extranjeros que contratados como profesores en las universidades formaron a las primeras generaciones de geógrafos latinoamericanos (Gangas y Santis, 1981).

Debido a ello, RGNG entre 1974 y 1990 se caracteriza, principalmente, por constituir un medio de difusión de investigaciones, tanto de los académicos de la institución patrocinante (del Instituto de Geografía de la Pontificia universidad Católica de Chile) como también de académicos nacionales filiados a otras universidades del país. Sin embargo, no deja de ser destacada una participación como autores principales o coautores de investigadores europeos, principalmente del mundo germano (13\% del total) y del Reino Unido, Francia y España (cerca del $2 \%$ ). A diferencia de la presencia de autores de origen europeo, la vinculación y, por tanto, la presencia de investigadores latinoamericanos, con la excepción de argentinos, es más bien inexistente (Figura $\mathrm{N}^{\circ} 1$ ).

Durante este periodo, resultan sumamente destacables los abundantes ejemplos de trabajos interdisciplinarios, generándose fructíferos diálogos entre la Geografía, la Historia y la Antropología, en especial en los trabajos relacionados con la región boreal del país (i.e. desierto de Atacama, comunidades y pueblos indígenas, altiplano). Dicha situación cambia radicalmente con la posterior consolidación de la investigación en el Instituto de Geografía de la PUC ${ }^{7}$, ante lo cual la revista adquiere una clara predominancia de trabajos de corte geográfico, Ilegando al final

\footnotetext{
7 En 1968, el Departamento de Historia y Geografía se independiza de la Facultad de Filosofía y Ciencias de la Educación, en 1970 se crea el Instituto de Geografía y en 1971 se aceptaron los primeros estudiantes en ingresar a un plan de licenciatura, aunque provenían del programa de profesores secundarios. Recién será en 1975, cuando se seleccionan los primeros estudiantes que ingresan vía admisión normal y en 1977 se sanciona el plan y programas de estudios conducentes al título profesional de geógrafos (Santis y Gangas, 1982).
} 
del periodo a representar más del $85 \%$ de los trabajos publicados (Figura $\mathrm{N}^{\circ} 2$ ).

En cuanto a las temáticas abordadas, RGNG recoge los temas de investigación que a los profesores extranjeros interesaban y que transfirieron a sus primeros discípulos chilenos (Gangas, 1985; Manríquez, 2001 2002). Las investigaciones pertenecientes a la denominada geografía humana son las predominantes, en tanto los artículos relacionados con la geografía física representaron el $28 \%$, cartografía un $4 \%$ y otras ciencias afines como historia social, historia económica, arqueología u ordenamiento territorial, menos de un $3 \%$ (Figura $\mathrm{N}^{\circ} 2$ ). Un análisis por subdisciplinas geográficas revela que el mayor peso correspondía a los artículos de geomorfología $(18 \%)$, seguidos por geografía de la población y geografía histórica con 10,5\% cada una y teoría de la geografía $(9 \%)$. A partir del segundo tercio del periodo estudiado, comienzan a surgir nuevas temáticas que adquirirán gran importancia en la etapa siguiente: como geografía urbana, geografía política y enseñanza y didáctica de la geografía.

Figura $\mathrm{N}^{\circ} 1$

Procedencia de los artículos publicados en la Revista de Geografía Norte Grande según la filiación institucional de los autores principales entre 1974 y 1990

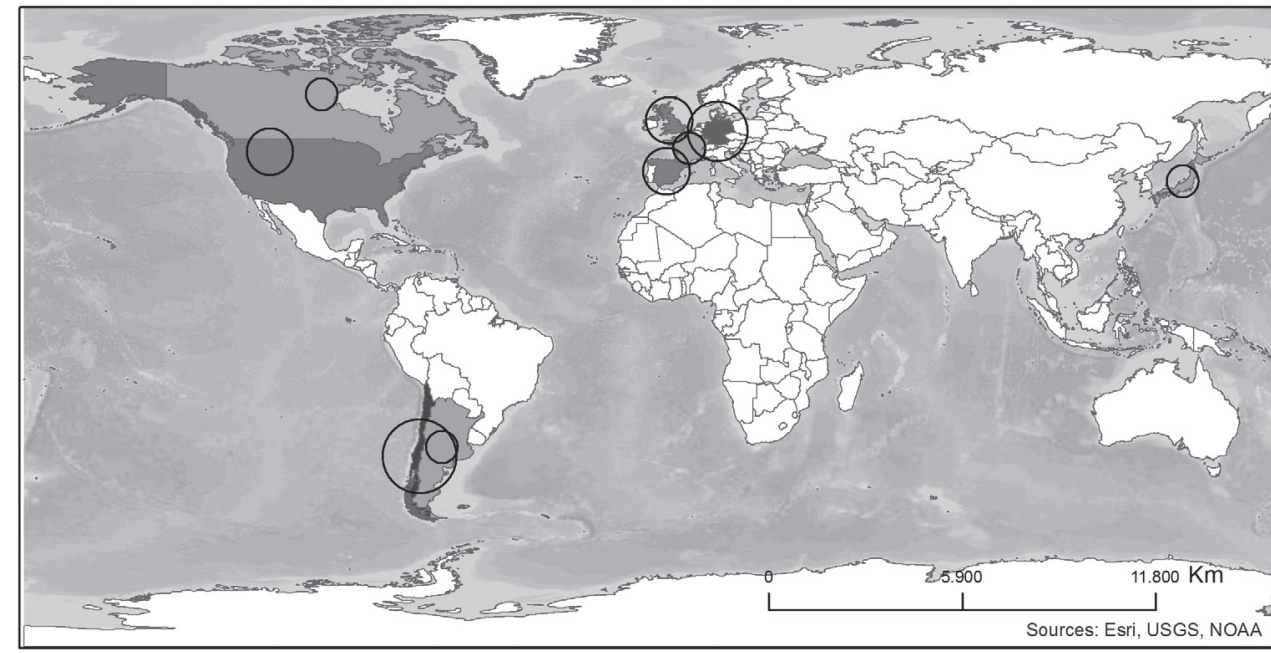

Leyenda

Participación porcentual 1974-1990 Artículos publicados 1974-1990
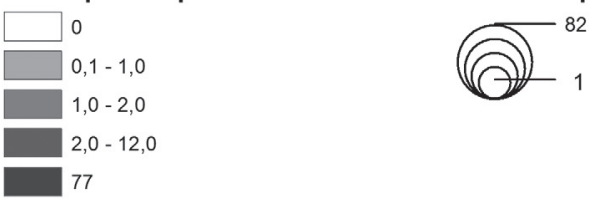

Fuente: Elaboración propia.

Las palabras clave utilizadas por los autores en sus artículos entre 1974 y 1990 (Figura $N^{\circ} 3$ ) constituyen un aspecto muy interesante, pues reflejan el interés de los investigado- res por ir construyendo un bagaje conceptual más sólido por parte de la geografía, así como el ir posicionando nuevas temáticas. 
Figura $\mathrm{N}^{\circ} 2$

Procedencia disciplinar de los artículos publicados en la Revista de Geografía Norte Grande según la filiación institucional de los autores principales entre 1974 y 1990

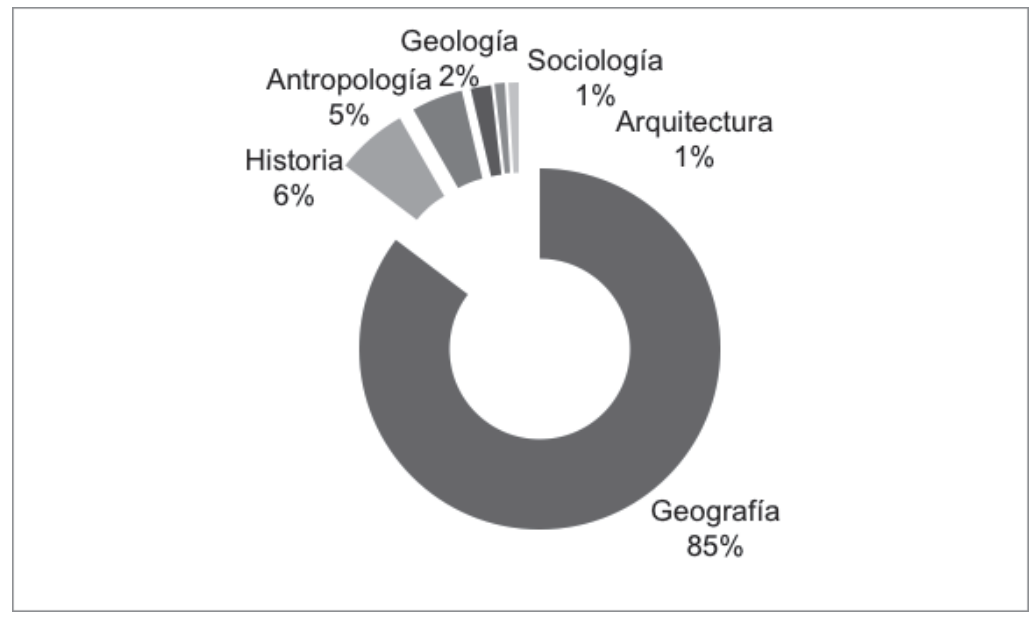

Fuente: Elaboración propia.

Figura $\mathrm{N}^{\circ} 3$

Palabras clave utilizadas por los artículos publicados en Revista de Geografía Norte Grande entre 1974 y 1990

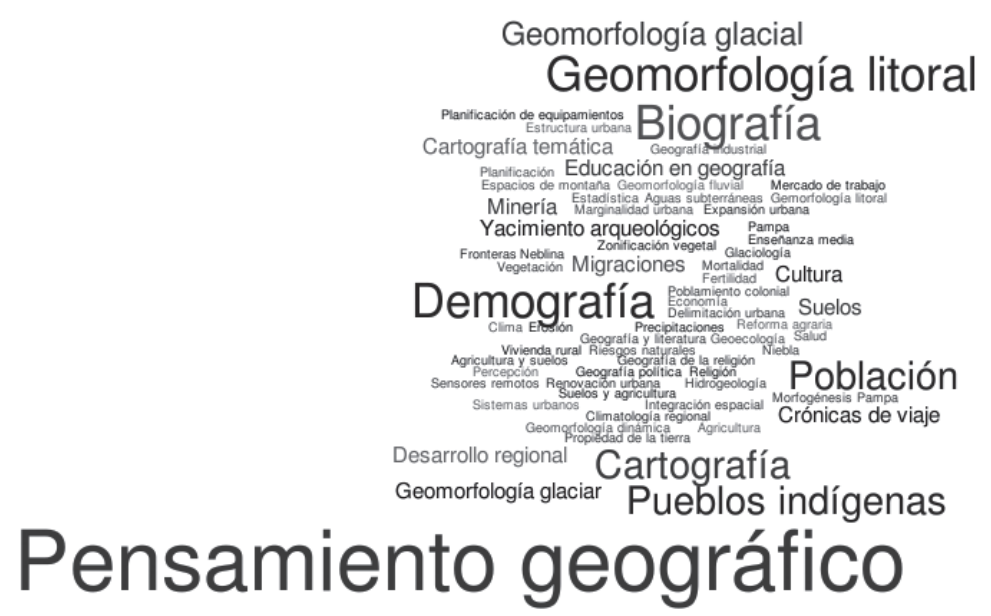

Fuente: Elaboración propia.

1991-2005: Nuevas ideas, nuevas geografías

El retorno a la democracia en Chile, la consolidación del proceso de neoliberalización y la intensificación de la globalización abrieron nuevos desafíos, tanto para la ciencia geográfica chilena como para Revista de Geografía Norte Grande. Aunque continuaron los tradicionales nexos con académicos de Argentina y del mundo germano, Francia surge como el país extranjero con mayor aporte de autores $(4 \%)$, preferentemente en el campo de la geografía física. A su vez, comienzan a aparecer autores provenientes de otros países "no tradicionales" en la revista: Brasil, Colombia, Costa Rica, Cuba, México, Estados Unidos, Níger y Australia (Figura $\mathrm{N}^{\circ} 4$ ). 
Figura $\mathrm{N}^{\circ} 4$

Procedencia de los artículos publicados en la Revista de Geografía Norte Grande según la filiación institucional de los autores principales entre 1991 y 2005

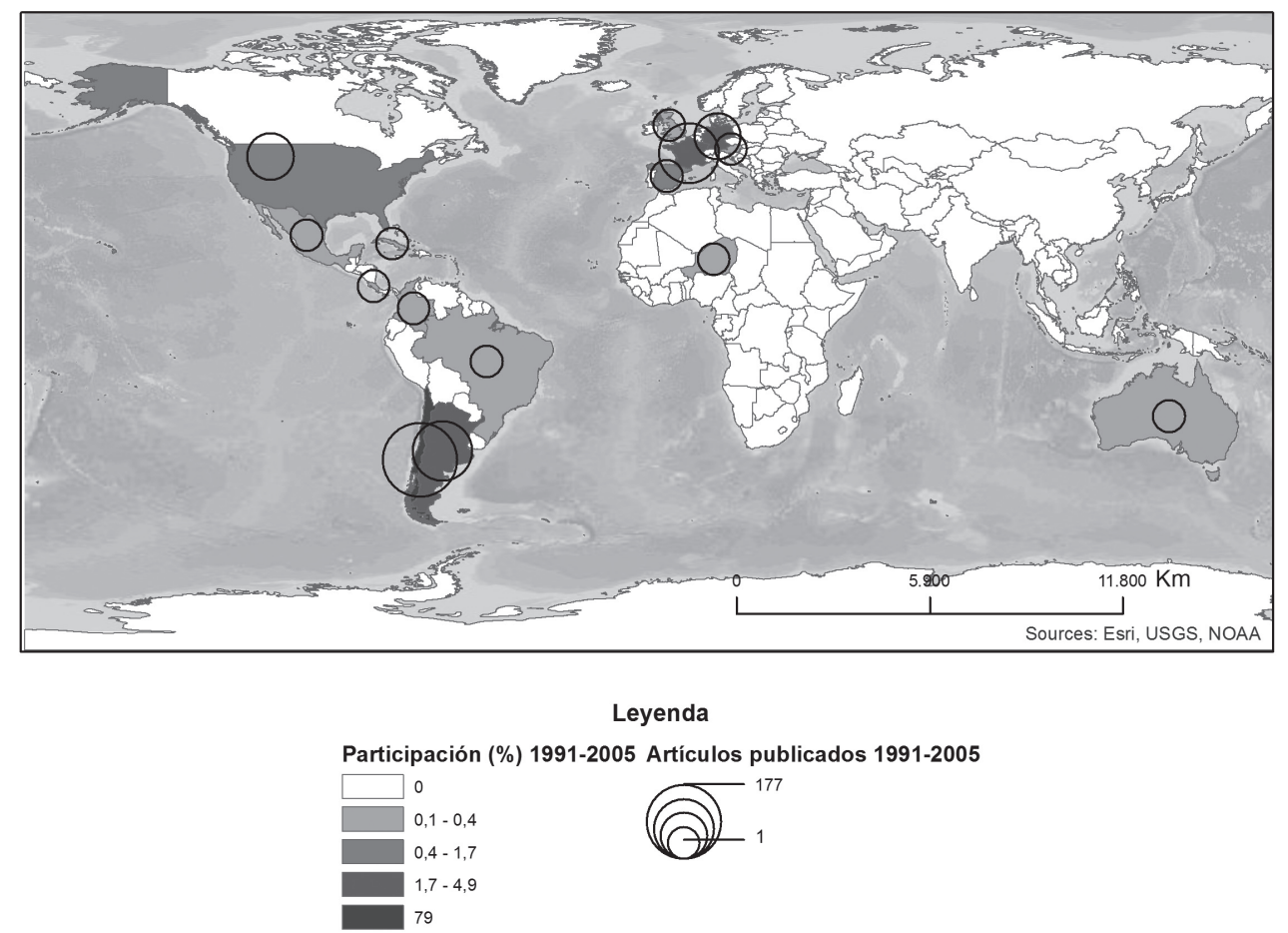

Fuente: Elaboración propia.

El carácter multidisciplinario se mantuvo estable respecto al periodo anterior registrándose la participación de siete disciplinas. Sin embargo, la presencia mayoritaria de estudios exclusivamente geográficos, y que ya había comenzado a destacar desde principios de la década de 1980, se consolida, siendo aún más notoria la presencia de académicos pertenecientes al Instituto de Geografía de la Pontificia Universidad Católica de Chile, los cuales son autores o coautores del 91\% del total de artículos publicados (Figura $N^{\circ} 5$ ).

Desde mediados de los años noventa del siglo pasado la "geografía vive y padece su propia condición postmoderna" (Delgado, 2003: 141) y la revista es reflejo de ello. De esta manera, coexisten numerosos enfoques, desde los neopositivistas fortalecidos por la masificación de las tecnologías digitales de la información geográfica, pasando por los neomarxistas aún dominantes en contextos como el latinoamericano hasta las geografías postmodernas y posestructuralistas que revalorizan lo local, lo subjetivo y lo diferente. La geografía humana adquiere un mayor dinamismo y riqueza teórica-conceptual, lo cual lleva a una creciente escisión con la geografía física. La primera durante este periodo concentra más del $57 \%$ de los artículos publicados, mientras que la segunda permanece estable con un $29 \%$.

A pesar de lo anterior, la geomorfología continúa siendo la subdisciplina de mayor participación con un 16\%, seguida en el campo de la geografía física, por la climatología con un 7\%. En cuanto a la geografía humana, el avance del proceso de urbanización a escala global y local se refleja en el 
Figura $\mathrm{N}^{\circ} 5$

Procedencia disciplinar de los artículos publicados en la Revista de Geografía Norte Grande según la filiación institucional de los autores principales entre 1991 y 2005.

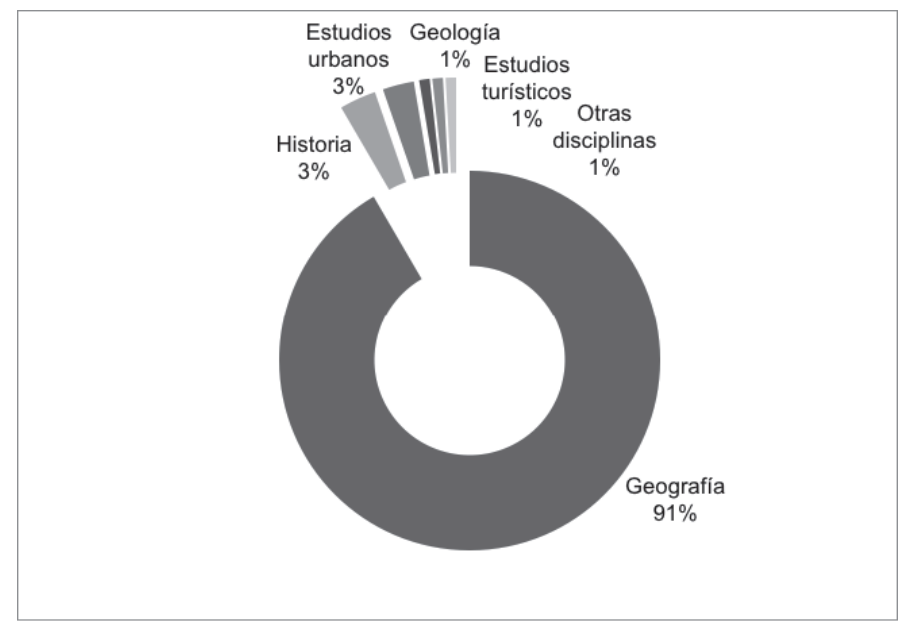

Fuente: Elaboración propia.

cuantioso aumento de artículos orientados a la geografía urbana (14\%), la geografía histórica $(9 \%)$ y la geografía económica $(6 \%)$. Aún más interesante, resulta ser el surgimiento de trabajos orientados a la planificación y el ordenamiento territorial que pasaron de representar menos del $1 \%$ entre 1974 y 1990 a 7,7\% en el lapso 1991-2005.
Lo anterior, queda muy bien reflejado en la Figura $N^{\circ} 6$ donde las palabras clave más utilizadas se refieren a pensamiento geográfico, ordenamiento territorial y ambiental, planificación urbana y desarrollo regional, ahora abordadas no solo desde la geografía humana sino que también desde la geografía física. A su vez, la mayor diversidad de palabras clave

Figura $\mathrm{N}^{\circ} 6$

Palabras clave en la Revista de Geografía Norte Grande entre 1991 y 2005

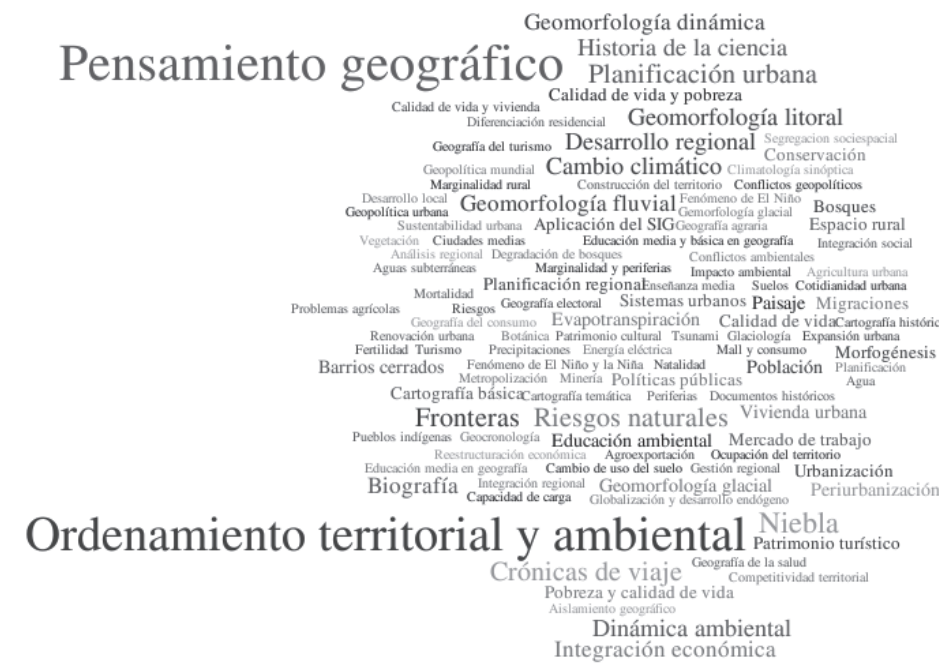

Fuente: Elaboración propia. 
utilizadas por los autores demuestra no solo la multiplicidad de perspectivas geográficas existentes, sino que también su cada vez mayor especialización.

\section{6-2014: Una creciente interdisciplinariedad e internacionalización}

En la última década, Revista de Geografía Norte Grande establece un plan de desarrollo destinado a lograr su internacioalización e indexación en las principales bases de datos y catálogos científicos. Así logra su ingreso a SciELO y Scopus en 2005 y a Web of Science (ex ISI, Thompson-Reuters) en 2009.

Lo anterior, rápidamente se refleja en el origen de los autores. Los pertenecientes a universidades chilenas descienden al 51\%, lo que contrasta con su alta concentración entre 1974 y 2006 (79\%). Mientras que, la nacionalidad de los autores demuestra su alcance Iberoamericano, distribuyéndose ente Argentina (13\%), España (9\%), México $(8 \%)$ y Brasil $(8 \%)$, es decir, RGNG adquiere claramente un alcance iberoamericano (Figura $N^{\circ} 7$ ).

Por otra parte, si en el periodo anterior más del $90 \%$ de los artículos producidos en instituciones chilenas se concentraban en Santiago, entre 2006 y 2014, dicha situación se ha visto radicalmente modificada gracias a una mayor participación de universidades regionales, localizadas en ciudades como Concepción, Temuco, Valdivia, Osorno y Punta Arenas (Figura $\mathrm{N}^{\circ} 8$ ), decayendo el vínculo existente con las universidades del norte de Chile (en Antofagasta y Arica).

\section{Figura $\mathrm{N}^{\circ} 7$}

Procedencia de los artículos publicados en la Revista de Geografía Norte Grande, según la filiación institucional de los autores principales entre 2006 y 2014

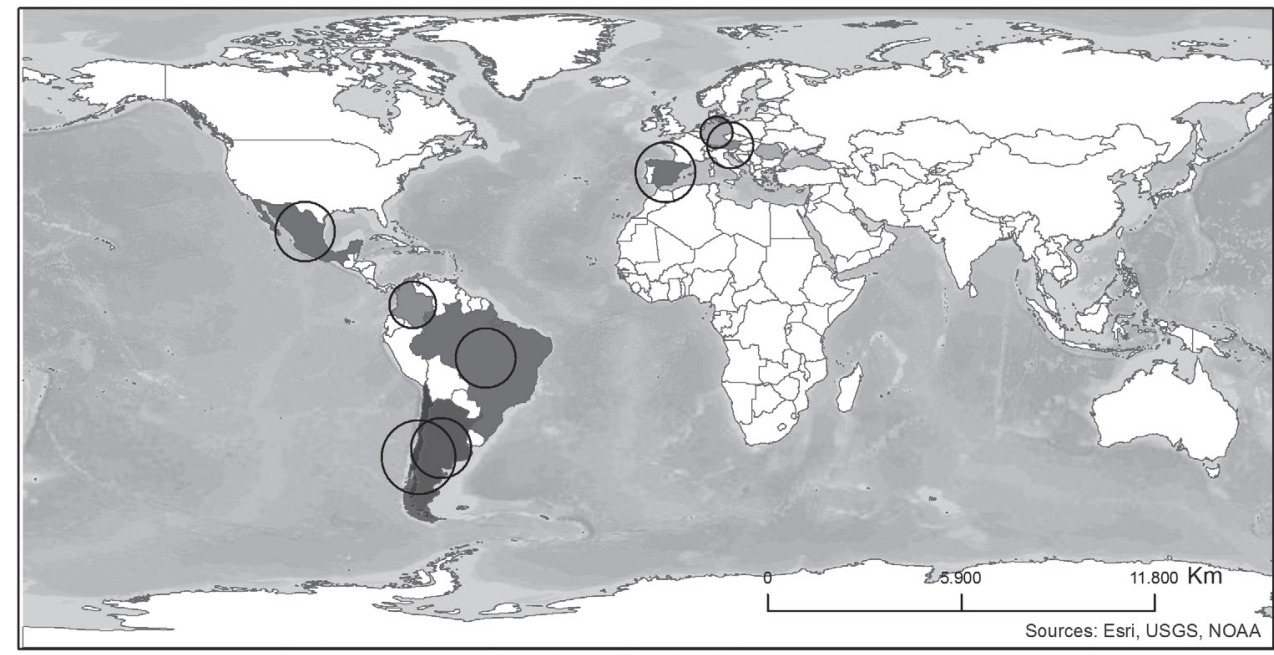

Leyenda

Participación porcentual 2006-2014 Artículos publicados 2006-2014
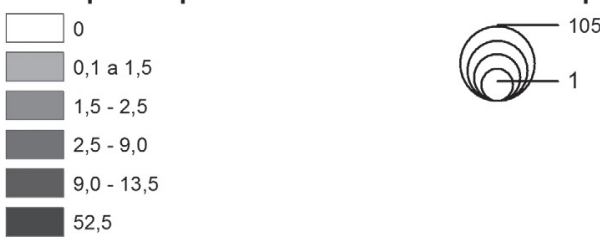

Fuente: Elaboración propia. 
Figura $N^{\circ} 8$

Procedencia de los artículos publicados chilenos en la Revista de Geografía Norte Grande, entre 1974 y 2014
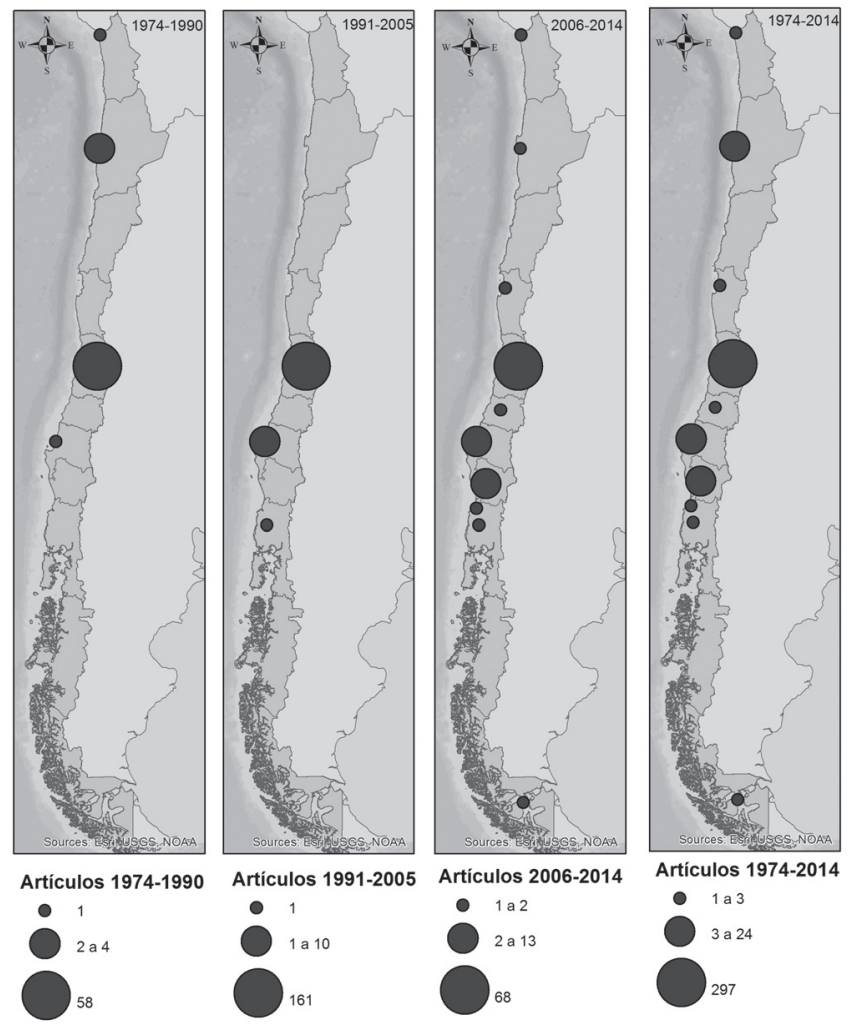

- 1 a 2

Artículos 1974-2014

2a 13

- 1 a 3

3 a 24

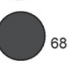

297

Fuente: Elaboración propia.

A diferencia de los periodos anteriores, entre 2006 y 2014, la revista acogió una mayor cantidad de artículos provenientes de diversas disciplinas, es decir la interdisciplinariedad se transforma en un paradigma. La participación de la geografía se reduce al $67 \%$, mientras que aumenta la presencia de las ciencias naturales y ambientales, como la biología y la ecología al igual que los estudios urbanos y turísticos (Figura $N^{\circ}$ 9).

En cuanto a las subdisciplinas, RGNG continúa publicando trabajos ligados a la geografía humana (51\%). Siendo ahora la geografía urbana, la línea con mayor presencia (21\%), desplazando a subdisciplinas que habían predominado en los dos periodos anteriores, como la geomorfología (13\%). Se destaca el surgimiento de trabajos relacionados con la geomática $(7 \%)$ y de las llamadas "nuevas geografías" relacionadas con turismo, violencia, representación, entre otros temas (5\%).

Las palabras clave de los últimos años revelan la continuidad del interés reflexivo del pensamiento geográfico (Figura $N^{\circ} 10$ ), una disciplina en perpetuo crecimiento, aunque a diferencia de periodos anteriores, también se presenta "más latinoamericana". En cuanto a las temáticas más recurrentes destacan las dinámicas ambientales, riesgos naturales, climatología urbana y geomorfología fluvial. Mientras que las problemáticas urbanas están presentes a través de conceptos como gentrificación, segregación social, metropolización y migración. 
Figura $N^{\circ} 9$

Procedencia disciplinar de los artículos publicados chilenos en la Revista de Geografía Norte Grande, entre 2006 y 2014

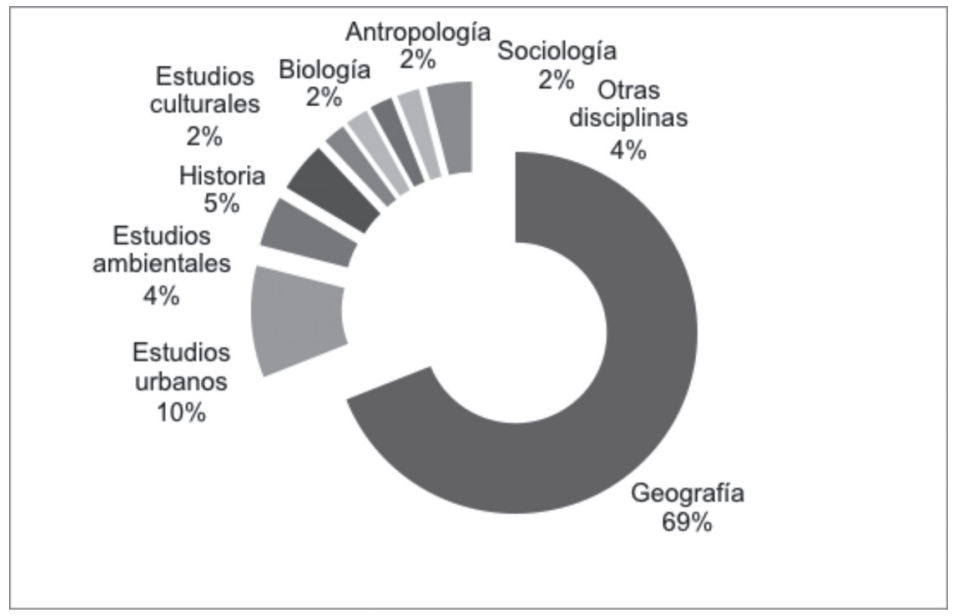

Fuente: Elaboración propia.

Figura $\mathrm{N}^{\circ} 10$

Procedencia de los artículos publicados en la Revista de Geografía Norte Grande, según la filiación institucional de los autores principales entre 1974 y 1990.

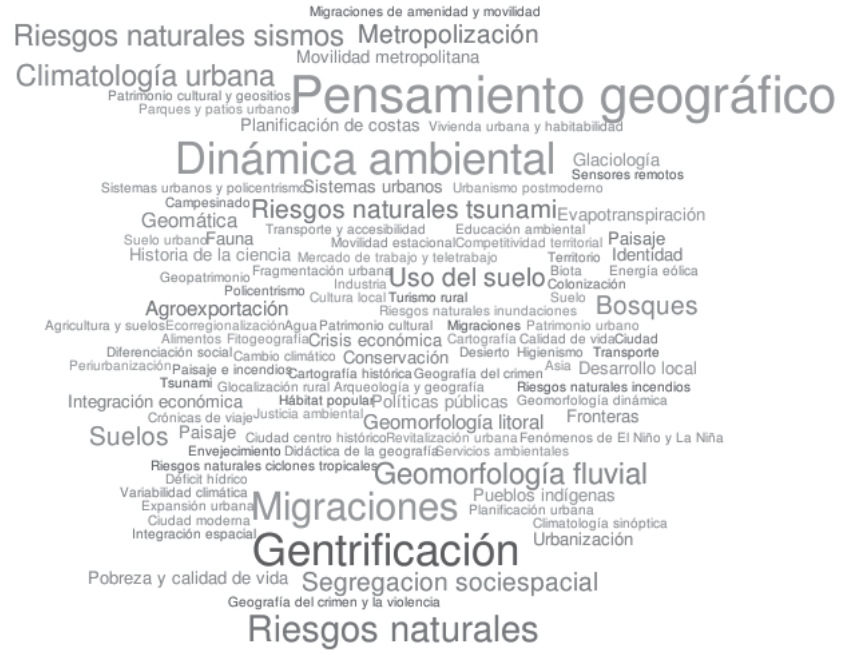

Fuente: Elaboración propia

\section{Reflexiones finales}

Los artículos publicados de RGNG entre 1974 y 2014 reflejan adecuadamente lo que ha ocurrido en la ciencia geográfica elaborada en América Latina y el Caribe. Hasta me- diados de los años ochenta del siglo pasado, la Geografía, salvo en casos excepcionales, aún bebía del aporte que realizaron una serie de profesores extranjeros, especialmente provenientes de Europa occidental y de Estados Unidos de América, que arribaron en 
los distintos países latinoamericanos durante esos años, colaborando en la conformación de Institutos y Departamentos de Geografía -y ciencias afines- en las universidades, y formando y perfeccionando a los primeros profesionales y docentes de las carreras de Pedagogías que se interesaban por el estudio de los territorios.

Posteriormente, en la última década del siglo pasado, los nuevos desafíos que implicaban el derrumbe de la Unión Soviética, la expansión del modelo económico neoliberal y el acelerado proceso de globalización sumado a la reactivación del crecimiento económico y una importante reducción de la pobreza por parte de los países latinoamericanos derivó en un giro y apertura de los intereses investigativos por parte de los geógrafos. De esta manera, nuevas problemáticas e interrogantes debían ser respondidas, lo cual se aunaba con el incremento de académicos que lograban acceder a programas de magíster y doctorado en el extranjero, quienes al volver traían nuevas perspectivas teóricas y herramientas metodológicas.

Los primeros años del presente siglo, no han detenido y ralentizado las transformaciones, sino que todo lo contrario, se han dinamizado producto de lo que algunos han denominado como una compresión espaciotemporal (Harvey, 1989). A su vez, el conocimiento contemporáneo presenta ahora como características, un crecimiento acelerado, una mayor complejidad y una rápida tendencia a la obsolescencia. De este modo, en las universidades y centros de investigación se reduce el tiempo de graduación y de posgraduación, el número de investigadores con grado de doctor se anhela y multiplica, la docencia gira hacia una rápida transferencia de conocimiento y adiestramiento de profesionales y el éxito de los académicos se mide en productividad demostrable y cuantificable (Tünnerrnann Bernheim y de Souza Chaui, 2003). Lo anterior, se ha reflejado en un incremento de nuevas perspectivas, temáticas y metodologías, muchas de ellas surgidas en Latinoamérica y que demuestran el desarrollo y consolidación que la ciencia geográfica comienza a mostrar en esta parte del mundo.

Lo anterior, exige, hoy en día, que las publicaciones seriadas tengan un manejo más profesional, eficiente y oportuno del inmenso conocimiento científico generado. Un desafío inmenso, y que Revista de Geografía Norte Grande ha intentado e intentará responder de la mejor forma posible.

\section{Referencias bibliográficas}

ASOCIACIÓN AMERICANA DE GEÓGRAFOS (AAG). El estado actual de la geografía en los países hispanoamericanos. Chicago: Panel organizado por la Asociación Americana de Geógrafos (AAG) y El Grupo de Especialidad Latinoamericanista Reunión de la Asociación Americana de Geógrafos, 2006. Disponible en Internet: http://www.aag.org/ galleries/project-programs-files/el_estado_actual_de_la_geografia.pdf

ABADAL, E. y RIUS, LL. Revistas científicas digitales: características e indicadores. Revista de Universidad y Sociedad del Conocimiento, 2006, Vol. 3, No 1, p. 6-20.

ANCKER, J. Proceedings of the retreat on The Journal's Role in Scientific Misconduct. Virginia: A Council of Science Editors retreat with funding from the Office of Research Integrity, 7-9 November 2003, Lansdowne Resort and Conference Center Lansdowne, 2003. Disponible en Internet: http://ori.hhs.gov/sites/default/files/editor_retreat.pdf

BAHRENBERG, G. La geografia alemana, 10 años después de Kiel. De la antropogeografía a la investigación regional; un balance intermedio. Geocrítica, Cuadernos Críticos de Geografía Humana, 1979, Año 4, № 24. Disponible en Internet: http://www.ub.edu/ geocrit/geo24.htm

BRIGNARDELLO, L. Índice temático de los trabajos publicados en los números 5 al 22 de la Revista de Geografía Norte Grande (1976-1996). Revista de Geografía Norte Grande, 1996, №23, p. 113-118.

DELGADO, O. Debates sobre el espacio en la geografía contemporánea. Bogotá: Universidad Nacional de Colombia, 2003.

GANGAS, M. y SANTIS, H. Notas para un análisis crítico del desarrollo de la Geografía en Chile. Revista Geográfica de Chile Terra Australis, 1981, N²5, p. 75-78. 
GANGAS, M. Los temas de investigación práctica en la Geografía Chilena (18301980). Revista de Geografía Norte Grande, 1985 , No 12 , p. 49-63.

GUTIÉRREZ PUEBLA, J. Las revistas internacionales de geografía: internacionalización e impacto. Boletín de la Asociación de Geógrafos Españoles, 1999, № 27, p. 117-134.

HARVEY, D. The condition of postmodernity: an enquiry into the origins of cultural change. Cambridge: Blackwell, 1989.

MANRÍQUEZ TIRADO, H. Los temas de investigación geográfica en Chile a través de sus revistas. Revista Geográfica de Chile Terra Australis, 2001-2002, №32-33, p. 241-247.

MOREIRA, R. O pensamento geográfico brasileiro. As matrizes originárias. São Paulo: Editora Contexto, 2008.

PALACIO PRIETO, J.L. Los estudios de Geografía en las universidades de América Latina; desarrollo, situación actual y perspectivas. Investigaciones Geográficas, Boletín del Instituto de Geografía, UNAM, 2011, № 74, p. $107-124$

PEET, R. Una nueva geografía de izquierdas. En: BENACH, N. (editora). Richard Peet. Geografía contra el neoliberalismo. Barcelona: Icaria, 2012, p. 91-94.
RED DE INDICADORES DE CIENCIA Y TECNOLOGÍA -IBEROAMERICANA E INTERAMERICANA (RICYT). Indicadores de internacionalización de la ciencia y la tecnología. 2015. Disponible en Internet: http://www. ricyt.org

SANTIS, H. y GANGAS, M. Notas para la historia de la geografía contemporánea en Chile (1950-80). Revista de Geografía Norte Grande, 1982, Vol. 16-17, № 1, p. 5-17.

TÜNNERMANN BERNHEIM, C. Y DE SOUZA CHAUI, M. Desafíos de la Universidad en la Sociedad del Conocimiento, Cinco Años Después de la Conferencia Mundial sobre Educación Superior. París: Comité Científico Regional para América Latina y el Caribe del Foro de la UNESCO, 2003.

ZAPATER, E.; ANDRADE, B. y ARAYA VERGARA, J.F. Índice temático de trabajos publicados en los números 1 al 25 de Informaciones Geográficas (1949-1978). Informaciones Geográficas, 1978, № 25, p. 77-81. 